\title{
Assessment of the Cardiovascular Risk and Physical Activity of Individuals Exercising at a Public Park in the City of São Paulo
}

\author{
Cláudia L. M. Forjaz, Taís Tinucci, Teresa Bartholomeu, Tiago E. M. Fernandes, \\ Vivian Casagrande, José Geraldo Massucato
}

São Paulo, SP - Brazil

\begin{abstract}
Objective - Physical exercise helps to prevent cardiovascular disorders. Campaigns promoting exercise have taken many people to the parks of our city. The most appropriate exercise for preventing cardiovascular disorders is the aerobic modality; inappropriate exercise acutely increases cardiovascular risk, especially in individuals at higher risk. Therefore, assessing the cardiovascular risk of these individuals and their physical activities is of practical value.
\end{abstract}

Methods - In the Parque Fernando Costa, we carried out the project "Exercício e Coração" (Exercise and Heart) involving 226 individuals. Assessment of the cardiovascular risk and of the physical activity practiced by the individuals exercising at that park was performed with a questionnaire and measurement of the following parameters: blood pressure, weight, height, and waist/hip ratio. The individuals were lectured on the benefits provided by exercise and how to correctly exercise. Each participant received a customized exercise prescription.

Results - In regard to risk, $43 \%$ of the individuals had health problems and $7 \%$ of the healthy individuals had symptoms that could be attributed to heart disorders. High blood pressure was observed in a large amount of the population. In regard to the adequacy of the physical activity, the individuals exercised properly. The project was well accepted, because the participants not only appreciated the initiative, but also reported altering their exercise habits after taking part in the project.

Conclusion - Data obtained in the current study point to the need to be more careful in assessing the health of individuals who exercise at parks, suggesting that city parks should have a sector designated for assessing and guiding physical activity.

Key words: cardiovascular risk, exercise, public park

Escola de Educação Física e Esporte da USP e Parque Fernando Costa

Mailing address: Cláudia Lúcia de Moraes Forjaz - Av. Prof. Mello Moraes, 65 05508-900 - São Paulo, SP, Brazil - E-mail: cforjaz@usp.br

English version by Stela Maris C. e Gandour
Cardiovascular disorders are the major cause of death in the adult population of our country ${ }^{1}$. The populations mainly affected by these disorders are those living in the major urban centers, such as the city of São Paulo. Due to the great relevance of these diseases, a lot of effort has been put into avoiding them, and this can be seen in the determination and control of the cardiovascular risk factors, such as sedentary lifestyle.

Currently, regular exercise practice has been well established to help in the prevention of and rehabilitation from cardiac diseases ${ }^{2-4}$. Individuals with a sedentary lifestyle who slightly increased the amount of exercise in their lives markedly reduced their cardiovascular risk ${ }^{2,4}$; this was due to the beneficial effects of exercise on the cardiovascular system ${ }^{5,6}$ and to the control of other risk factors ${ }^{6-8}$.

However, it is worth emphasizing that some exercises are more effective than others in promoting cardiovascular health. Currently, it has been shown that resistive exercises may produce beneficial cardiovascular effects; the benefits obtained with aerobic exercises, however, are more expressive. The aerobic exercises, ie, the activities involving large muscle groups, which are moved in a cyclic manner with low to moderate intensity, long duration, and performed 3 to 5 times a week, are the most recommended for primary and secondary cardiovascular disease prevention ${ }^{9}$.

Despite the confirmed benefits provided by physical activity, a large part of the population of the city of São Paulo, approximately $69 \%$ of the adults ${ }^{10}$, has a sedentary lifestyle. Therefore, health care-related entities have proposed campaigns ${ }^{11}$, such as Agita São Paulo, to motivate the population to become more active. The result is a large number of people exercising at the parks of the city. However, it is worth emphasizing that inappropriate physical exercise may result in an immediate increase in cardiovascular risk, because the risk for myocardial infarction in an individual with a sedentary lifestyle is 6 times greater during intense exercise than that at rest ${ }^{12}$. In addition, most parks do not have a sector for assessing and guiding physical activity.

Even though this initiative to promote physical activi- 
ty is significant and necessary from the public health point of view, we need to assess whether people who are exercising at the parks of the city have a risk compatible with unsupervised exercise practice and whether the activity being performed is adequate.

Therefore, this project aimed to assess the cardiovascular risk (checking the presence of diseases, risk factors, and cardiovascular symptoms) and the practice of physical activity by people exercising at the Parque Fernando Costa (Parque da Água Branca). In addition, this project also aimed an educational effect, instructing those at the park about the potentialities of the exercises in preventing cardiovascular disorders and the correct way to practice them. And finally, we tried to assess the effectiveness of this educational proposal.

\section{Methods}

The project "Exercício e Coração" was carried out during 4 weekends from October to November 2000 at the Fernando Costa Park in the city of São Paulo, in the state of São Paulo. This park is characterized as being strictly urban, located close to the central region of the city of São Paulo, and provides the local population with space for walking, running, and activities related to animal breeding and plant cultivation. This project consisted of 10 sessions per weekend, with 6 people attending each session, and a total of 240 assisted people. Each session was conducted by 5 monitors. Participation was free of charge, and anybody at the park could take part in the project, which was advertised in banners scattered throughout the park and through direct verbal contact.

The 4 major objectives of the project were as follows: 1) to assess the presence of risk factors in the population visiting the park; 2) to analyze the physical activity performed by that population; 3 ) to instruct the population about the correct practice of physical activity, with assessment of the effectiveness of this educational practice; and 4) to assess the receptivity of the project by those exercising at the park.

The sessions consisted of 3 parts. In the first 20 to 25 minutes, the risk factors and health problems were assessed. Then, a 20- to 25-minute lecture was offered about the benefits of exercise for health and the correct way to exercise. In the last 10 to 20 minutes, individualized exercise prescriptions were provided.

Assessment of the risk factors and health problems was performed with a questionnaire, which was based on the concepts of the American College of Sports Medicine (ACSM) and the American Heart Association (AHA) ${ }^{13}$. The questionnaires were completed by the participants with the help of a monitor. The initial questions were about the presence of health problems, use of medication, and the presence of symptoms that could reflect cardiovascular disorders, such as chest pain, dyspnea, dizziness, and fainting. The second part of the questionnaire comprised questions about the presence of risk factors, such as sex, age, heredity, diabetes, and smoking; the participants were also asked whether they knew their glucose and serum cholesterol levels.

To assess the presence of obesity, weight and height were measured (Filizola). The waist and hip circumferences were also measured with a metric tape.

Blood pressure was measured with an aneroid sphygmomanometer 1 single time after a 5-minute sitting rest with the arm positioned at the level of the left ventricle. These measurements were taken by trained monitors. The I and V Korotkoff phases were used to determine the systolic and diastolic blood pressures, respectively. The sphygmomanometers calibration were constantly assessed against the mercury column during the project. To avoid measurements falsely reduced by physical activity prior to the evaluation, the participants were asked whether they had exercised prior to the session.

The questions about physical activities were as follows: which activities were performed, how many times a week, for how long, and an assessment about the intensity, which was classified as mild, moderate, or intense. The activity was considered mild if the individual did not feel significant modifications after the activity; moderate if the individual reported sweating and fatigue after the activity; and intense if the individual reported shortness of breath at the end of the activity. The last question asked permission to use the data for publication. The questionnaires were dated and signed by the participants.

The lessons were given by the previously trained monitors. Posters were used in these lessons. Each lesson comprised 5 theoretical topics: 1 ) the importance of cardiac disease; 2) definition of coronary disease; 3 ) risk factors of cardiac disease; 4) benefits of exercise; and 5) the most appropriate physical activity. Each lesson also comprised a practical topic, which consisted of teaching how to measure heart rate by palpation of the radial pulse, and how to use this measurement to assess the intensity of the exercise.

Based on data from the questionnaire, each participant received an assessment of his/her individual cardiovascular risk, using the North-American table, and an individualized prescription for walking or running. The prescription contained the appropriate week frequency for walking or running, the adequate duration of the activity, and, mainly, the range of heart rate that should be maintained during the activity. For prescription, we used the software of the CD-ROM that accompanied the book Risco Cardiovascular Global ${ }^{14}$, which was developed by 1 of the authors. This range was determined based on the age for the healthy individuals who did not undergo a recent exercise test. For the individuals with heart diseases or who were on medications that interfered with heart rate, only those with an exercise test received a prescription. The individuals with many risk factors or symptoms were instructed to look for a physician, as were those with high blood pressure levels.

In addition, the individuals received instructions and a brochure about the requirements for the safe practice of exercise, such as clothes, food, places, times, and the cool down period. 
One to three months of the end of the project, we made telephone contact with the participants to assess the following: what they recalled from the project, whether the project had had any results, and how they evaluated the project. In regard to the given activities, they were asked what they recalled from the session at the park, and their answers were grouped into 8 categories as follows: 1) cardiac disease as a cause of death;2) coronary problems; 3 ) risk factors; 4) benefits of exercising; 5) most appropriate activity; 6) recommendations to be considered; 7) how to assess heart rate; and 8) their training frequency or risk assessment. In regard to the effectiveness of the program, the participants were asked whether they had changed their physical activity habits after the project, and, in the affirmative case, what had changed. They were asked whether they had tried to measure their heart rate, and whether they had been successful; whether they had passed the information received to other people, and whether they intended to continue following the recommendations provided. In regard to the evaluation of the project, the participants were asked what they had found about the project and whether they would like to repeat the assessment in the future.

The results were presented only in a descriptive form with the percentages calculated as a function of the number of responses for each item, because some participants did not answer all the questions.

\section{Results}

The project comprised 227 individuals, corresponding to $95 \%$ of the total project openings. Of these individuals, only 1 did not allow his answers to be used in the study; therefore, data here presented correspond to 226 participants.

The final sample comprised $126(56 \%)$ females and 100 (44\%) males (fig. 1), most of whom were 40 years old or older (mean age $=54.5 \pm 13.8$ years). Of these, 17 had cardiac problems, 72 had other health problems, and 137 had no health problems. It is worth noting that of the 137 individuals with no health problems, 9 were on medications, which were specific for cardiac problems in 3 individuals and antihypertension in 6 . Therefore, the total number of individuals with heart disease was $20(9 \%)$, and the number of individuals with health problems was 78 (34\%). In addition, of the 128 (57\%) remaining individuals who had no health problem and who were not on any medication, $21(16 \%)$ frequently complained of symptoms, such as dyspnea, chest pain, dizziness, and fainting. Therefore, only 107 individuals ( $47 \%$ of the total) had no health problems and no symptoms at all, and, thus, could be considered apparently healthy.

In regard to the presence of risk factors and considering the entire group (fig. 1), 52\% of the individuals had close relatives with cardiac diseases, $10 \%$ were smokers, $29 \%$ were ex-smokers, and $10 \%$ had a sedentary lifestyle. In regard to glycemia, 12 individuals (6\%) were diabetic, 95 ignored their glycemia, and of the 112 who knew their glycemic levels, only 1 reported a high one. In regard to serum cholesterol levels, 74 individuals (34\%) did not know their levels

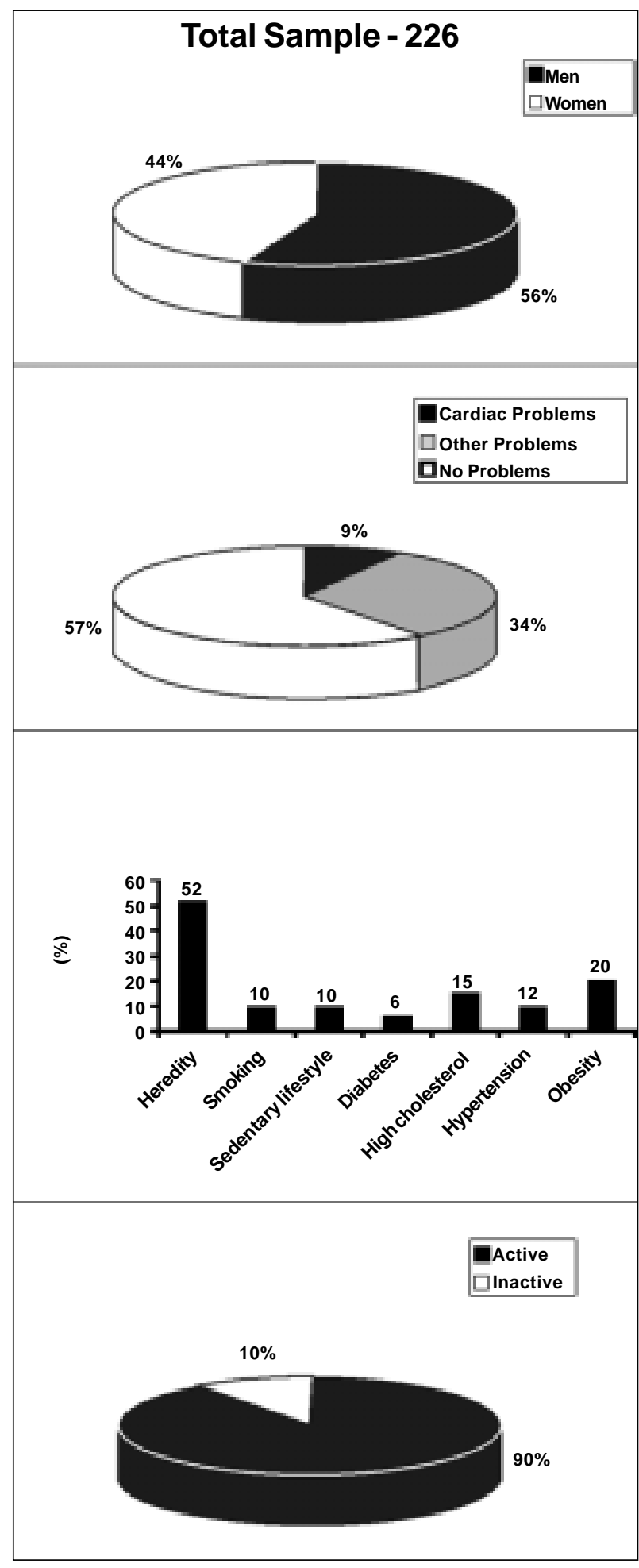

Fig. 1 - Characteristics of sex, presence of diseases and risk factors of individuals exercising at the Fernando Costa Park.

and $33(15 \%)$ reported high serum cholesterol levels. In regard to blood pressure, 22 individuals reported being hypertensive, and 6 were on antihypertensive medication, even though they did not consider themselves hypertensive. As such, the total hypertension rate was $12 \%$. However, it is noteworthy that of the 89 individuals who were not 
hypertensive and did not exercise prior to the session, 31 (35\%) had high levels when blood pressure was measured. In regard to obesity, $20 \%$ of the individuals had body mass index $>30 \mathrm{~kg} / \mathrm{m}^{2}$, and the waist/hip ratio of 7 males was $>1$ and of 47 females was $>0.85$.

Considering the practice of physical activity, 193 individuals (90\%) reported being active (fig. 1), walking being the most common activity, reported $77 \%$ of the time. In addition, most of the individuals practiced a physical activity 3 or more times a week $(66 \%)$, with a duration ranging from 30 to 60 minutes $(86 \%)$, and they considered that activity to be moderate $(63 \%)$.

Analyzing only the data referring to the 20 individuals with heart disease, 7 did not report which was the problem, 4 had valvular diseases, 3 had coronary artery disease, 2 had arrhythmias, 1 had Chagas' disease, and 3 used medication for cardiac diseases. Four (20\%) of these individuals reported frequent symptoms, such as dizziness, angina, and dyspnea. The mean age in the cardiac group was $60.9 \pm 13.7$ years.

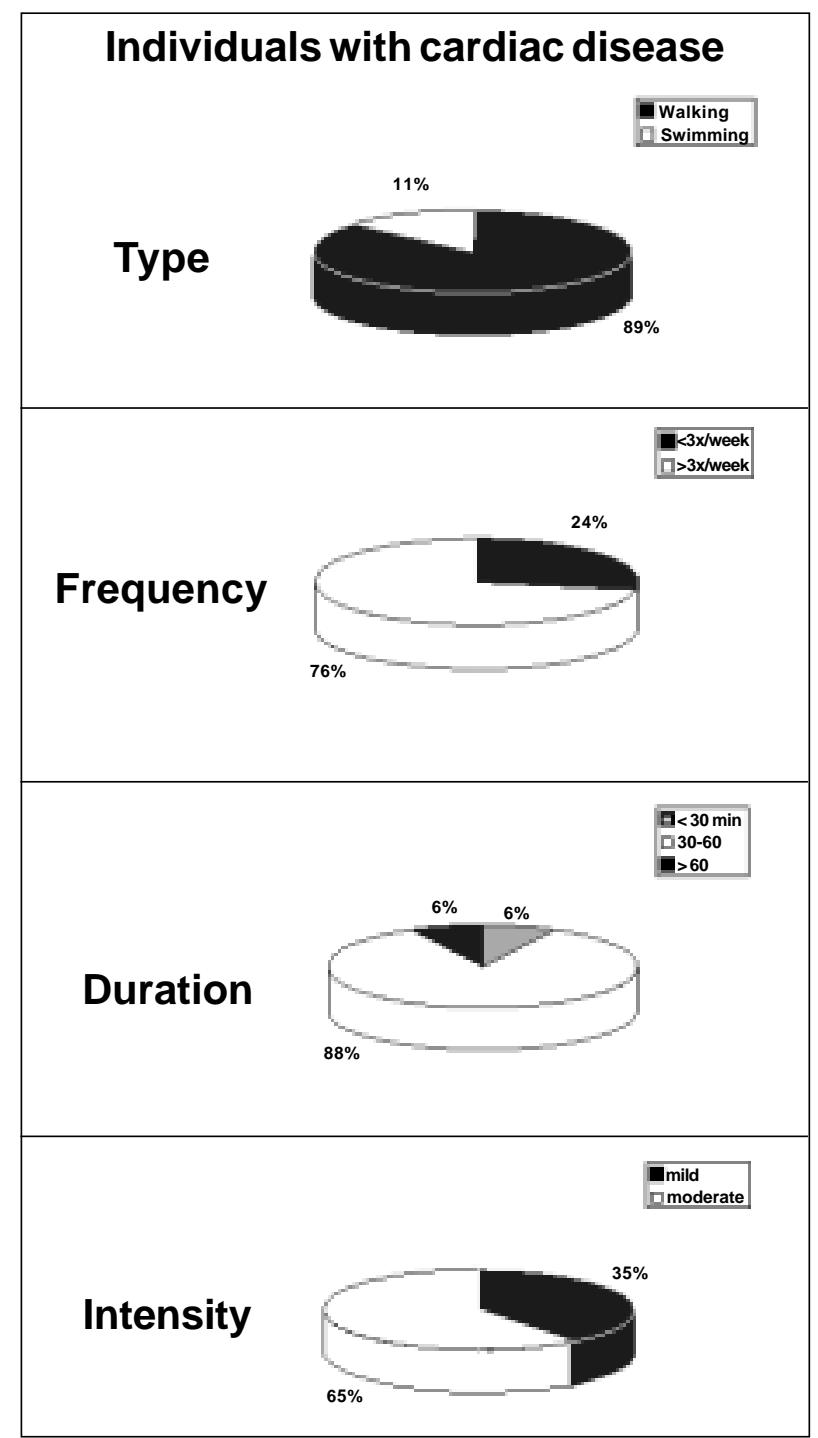

Fig. 2 - Characteristics of the physical activity practiced by individuals with heart diseases visiting the Fernando Costa Park.
In regard to the practice of physical activity, 5 (25\%) of these patients had received medical instruction to exercise, walking being the activity in 4 cases. Eighteen patients (95\% of those answering the questionnaire) reported practicing physical activity, walking being the major activity $(89 \%)$. In addition, they exercised 3 or more times a week $(76 \%)$, for 30 to 60 minutes per session $(88 \%)$, the intensity being mild in $35 \%$ and moderate in $65 \%$ (fig. 2 ).

Hypertension was the most prevalent health problem (12\%), affecting 17 females and 11 males. The mean age of the hypertensive individuals was $60.2 \pm 9.3$ years, and all of them were 40 or more years old. Of these hypertensive individuals, only 15 (54\%) were using some sort of medication. Only 17 hypertensive individuals had not exercised before the session, and, of these, 10 (59\%) had high blood pressure, of whom 5 had both high systolic and diastolic levels (fig. 3). Considering all the individuals, even those who had exercised prior to the session, 18 (64\%) had high blood pressure levels in the experimental session.

In regard to the practice of physical activity, $23(85 \%)$ patients reported exercising regularly, 20 of whom $(87 \%)$ walked (fig. 4). In addition, this activity was practiced 3 or more times a week $(70 \%)$ for 30 to 60 minutes $(83 \%)$ at a moderate intensity $(68 \%)$.

As already mentioned, 107 individuals had no known health problems, no symptoms, and used no medication, being, therefore, considered apparently healthy.

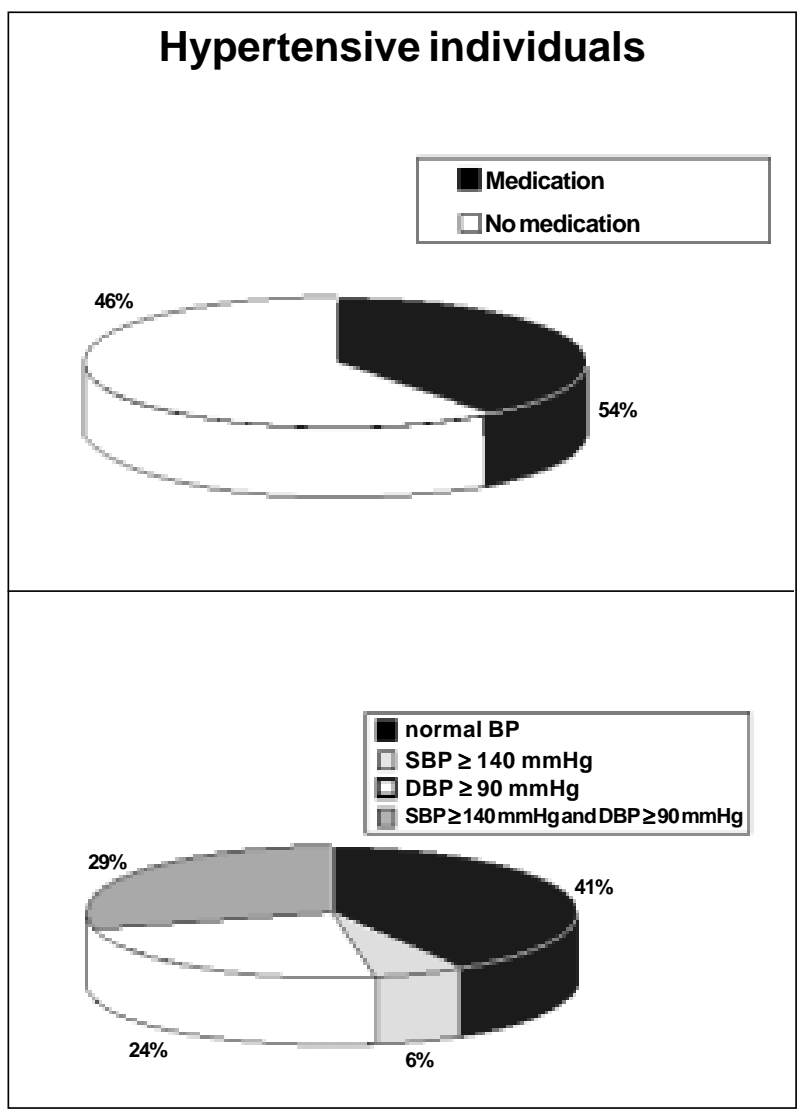

Fig. 3 - Blood pressure control of the hypertensive individuals exercising at the Fernando Costa Park. 


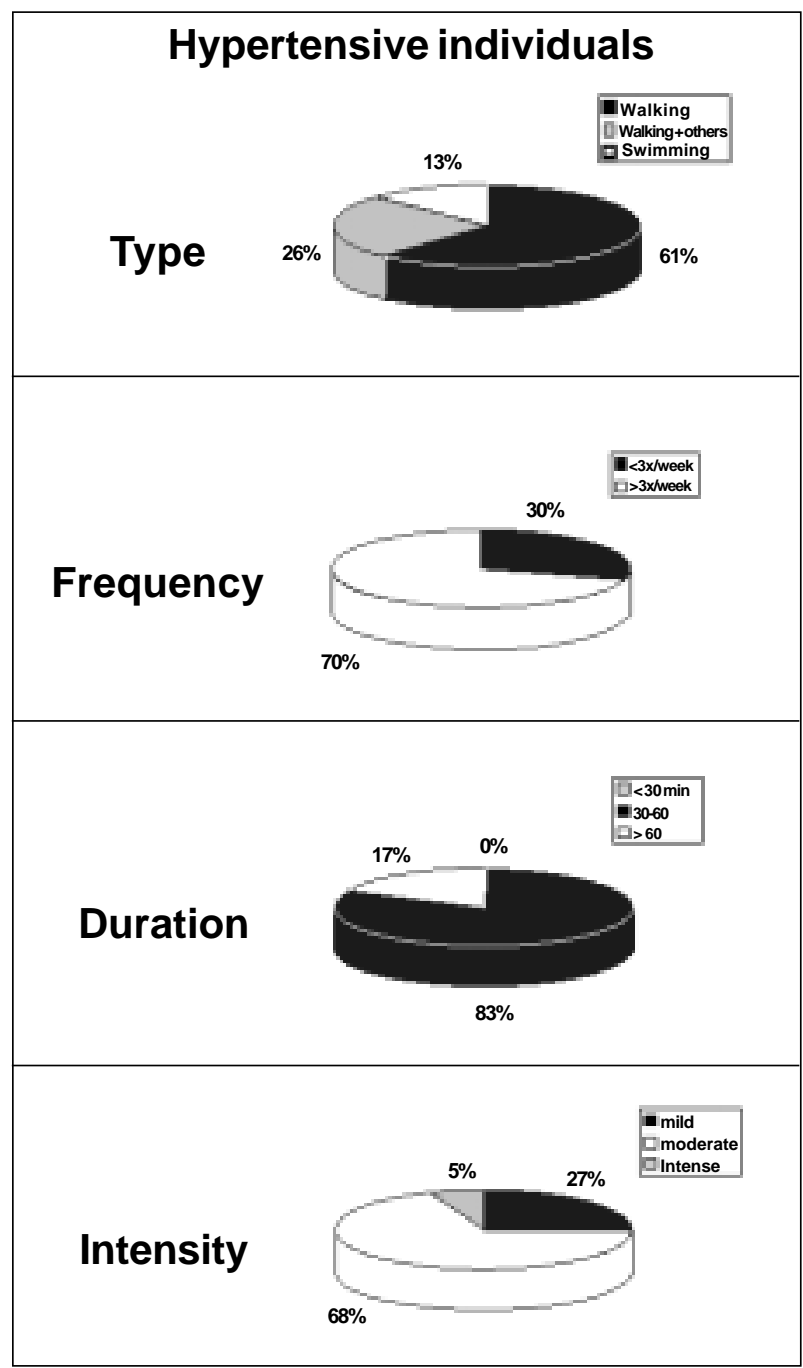

Fig. 4 - Characteristics of the physical activity practiced by the hypertensive visitors of the Fernando Costa Park.

Of these 107 individuals (fig. 5), 51 were males and 56 were females. The mean age in the group was $51.5 \pm 14.1$ years. Only 1 individual was less than 20 years old, 24 individuals were between 20 and 40 years of age, 48 were between 40 and 60 , and 34 were older than 60 years.

In regard to risk factors, $49 \%$ had close relatives with cardiovascular disease, $15 \%$ were smokers, $24 \%$ were exsmokers, and $8 \%$ had a sedentary lifestyle. In regard to glycemia, 50 individuals (49\%) ignored their levels and 52 (51\%) reported their glycemia as normal. In regard to serum cholesterol levels, 40 (40\%) individuals ignored their levels, $52(51 \%)$ reported normal serum cholesterol levels, and 9 (9\%) reported high serum cholesterol levels. In regard to blood pressure, 52 individuals had not exercised before the assessment; of these, 17 (33\%) had high blood pressure levels, $8(15 \%)$ of whom had both high systolic and diastolic levels. In regard to obesity, $14 \%$ of the individuals had a body mass index $>30 \mathrm{~kg} / \mathrm{m}^{2} ; 3$ males had waist $/$ hip ratio $\geq 1$, and 18 females had waist/hip ratio $\geq 0.85$.

In regard to the practice of physical activity (fig. 6), $92 \%$ of the individuals reported exercising regularly, wal-

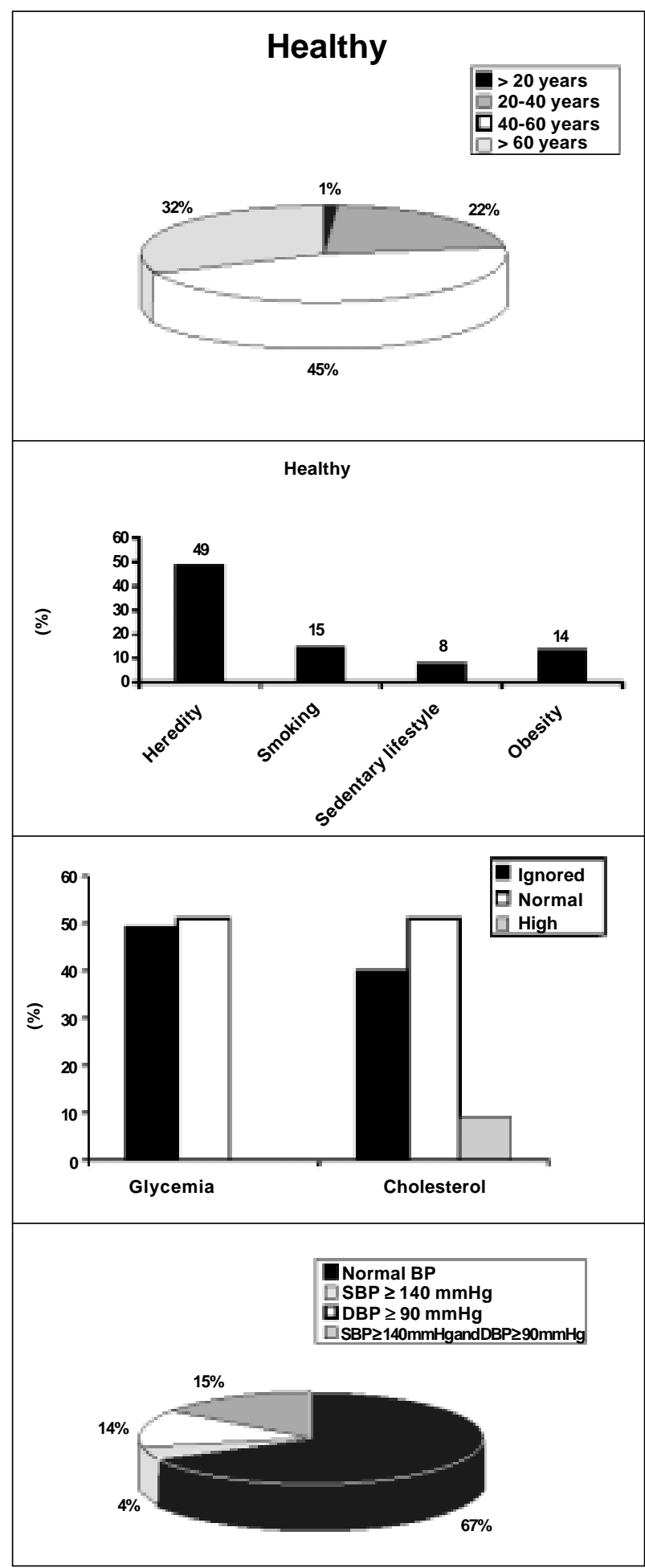

Fig. 5 - Characteristics of the apparently healthy population who visit the Fernando Costa Park.

king being the most common activity (73\%). The exercise was performed 3 or more times a week $(69 \%)$ for 30 to $60 \mathrm{mi}-$ nutes $(75 \%)$ with moderate intensity $(70 \%)$.

Of the 226 individuals taking part in the study, only 65 (29\%) could be contacted by telephone after the project. All of them reported recalling at least 1 of the topics of the theoretical lessons given, and the most recalled were the risk 


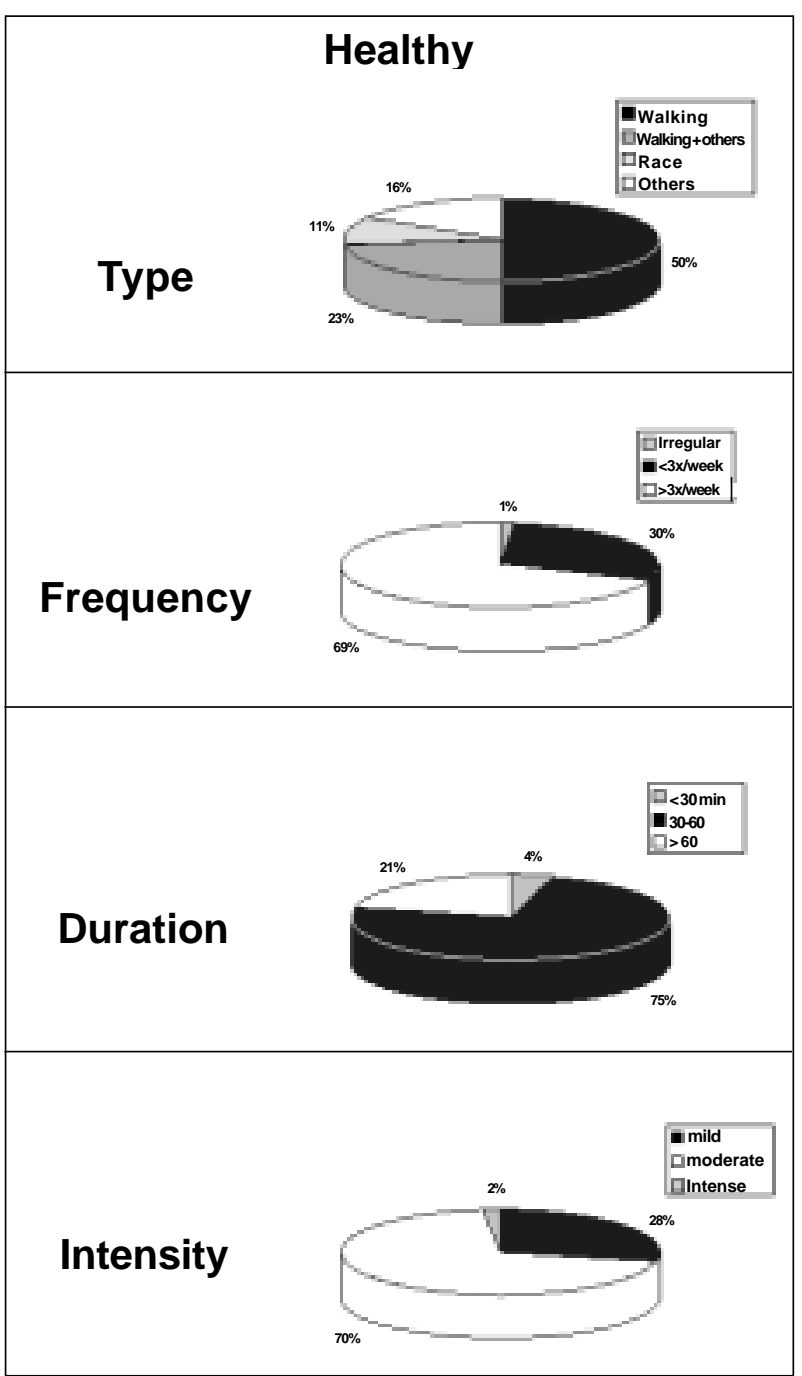

Fig. 6 - Characteristics of the physical activity practiced by the apparently healthy visitors of the Fernando Costa Park.

factors of cardiovascular diseases (26\%) and the benefits of exercising (26\%). In addition, 9 individuals recalled 2 topics and 4 individuals recalled 3 topics of the theoretical lessons. Twenty-five (39\%) individuals reported that the experience at the park modified their way of exercising; 3 of them initiated their practice, 7 changed the duration of the exercise, 7 changed the frequency, 7 altered the intensity, and 3 added a new activity to that already performed. After the experience at the park, $58 \%$ of the individuals reported being able to assess their heart rate during exercise, $17 \%$ reported not being able to, and $25 \%$ did not try to assess heart rate during exercise. In addition, $71 \%$ of the individuals reported informing other people about what they had learned in the park, and almost all of them (95\%) reported intending to follow the instructions they had been given. In general, 27 people rated the project as excellent, 29 as very good, 8 as good, and 1 as regular. In addition, $88 \%$ of the participants reported that they were interested in repeating the assessment in the future.

\section{Discussion}

The first objective of the project "Exercício e Coração" was to assess the cardiovascular risk of individuals exercising with no supervision at the parks of the city. We observed that $43 \%$ of the individuals exercising at the park had health problems ( $9 \%$ had heart diseases and $34 \%$ had other problems). In addition, it was evident that many individuals did not know their actual health condition, because $7 \%$ of the people who reported not having health problems were using cardiac medications, and, in addition, $16 \%$ of those who considered themselves healthy and used no medication had frequent symptoms. In fact, the presence of symptoms, such as dizziness, chest pain, and fatigue, may indicate the presence of cardiac problems ${ }^{9,13}$, increasing the risk of cardiovascular disorders, mainly during exercise ${ }^{9,12}$. These data suggest that we need to raise the consciousness of the population about their health, and, mainly, about the signs the organism sends indicating the need for looking for a doctor.

When the population with heart disease was considered, we observed that, even though physical exercise is extremely useful in cardiovascular rehabilitation ${ }^{6}$, only $29 \%$ of the patients had received a medical recommendation for that practice. It is worth emphasizing that a strong medical recommendation seems to be a fundamental factor for the patient's adherence to the practice of physical activity ${ }^{15}$. In addition, those receiving that recommendation reported that the doctor had only advised them to walk. However, moderate exercise in individuals with heart disease should be, at least initially, performed based on an ergometric assessment and under supervision, according to the recommendations of the ACSM/AHA ${ }^{13}$. It is also worth emphasizing that $20 \%$ of the individuals with heart disease reported symptoms during exercise, suggesting that these patients could be exercising under an ischemic condition, and, therefore, be at risk for arrhythmias and cardiac arrest ${ }^{9}$. The data presented indicate the need for a greater medical consciousness in regard to the recommendation of exercises for individuals with heart disease, and also the need for an appropriate recommendation, so that the patients know how to exercise at lower risk. In fact, the risk of individuals with heart disease exercising is lower in supervised programs ${ }^{16}$.

In regard to the presence of risk factors, we observed differences between the data of the general population of the municipality of São Paulo ${ }^{10}$ and those data obtained at the park. In addition to the methodological differences of measurement, the characteristics of the individuals who exercise at a park may explain these differences. For example, individuals who visit a park usually go there to exercise; therefore, only $10 \%$ of the individuals had a sedentary lifestyle in our sample, which was far below the $69 \%$ found for the city of São Paulo. Another interesting factor was the lower rate of smokers among those visiting the park, $10 \%$, as compared with 38\% found for the city of São Paulo. Even though no evidence of a direct effect of exercise on controlling smoking was observed, people who exercise tend to smoke less. In fact, the ex-smoker rate (29\%) was extremely 
high in our population. In the current study, we found a prevalence of obesity of $20 \%$ using as a cutpoint the body mass index value $>30 \mathrm{~kg} / \mathrm{m}^{2}{ }^{17}$. Rego et al ${ }^{10}$ found a similar prevalence with a lower cutpoint, suggesting that in our population obesity was higher. This difference may be related to the fact that obese people have been instructed to exercise, and, therefore, they may be more frequently found at parks. These data suggest that people go to parks to exercise, and this may be associated with other healthy behaviors, such as quitting smoking and trying to lose weight.

In regard to blood pressure, we observed that $12 \%$ of the individuals were hypertensive. However, of these individuals, approximately $60 \%$ had high blood pressure levels, suggesting that blood pressure was not under control, even though many of the individuals were on antihypertensive medication. In addition, 33\% of the apparently healthy population also had high blood pressure levels. Therefore, these data suggest that both blood pressure control and detection of hypertensive individuals are points requiring further study.

The second objective of this study was to assess the adequacy of the physical activity that has been spontaneously practiced by the population at the parks of the city. We could observe that walking was the most common activity, and this may have been influenced by the type of the park assessed, because the Parque Fernando Costa does not have facilities for other sports practices. Another point that may have had an influence was the fact that walking is the most easily performed type of exercise ${ }^{18}$. In regard to frequency, duration, and intensity, we observed that in all groups, most individuals exercised 3 or more times a week for 30 to 60 minutes at a moderate intensity. In fact, according to the American College of Sports Medicine ${ }^{9}$, this is the most appropriate recommendation for physical activity to improve health. These data show that most of the population visiting the park is exercising correctly, which results in low risk during the physical activity and in the possibility of obtaining cardiovascular benefits with exercise. Despite this adequacy, we observed that the control of the intensity of exercise was performed in a subjective way, because most people did not know how to measure their pulse.

The remaining objectives of this project were to assess, in a subsequent phase, its educational effect and its acceptability by the population assisted. We could observe that the project was well accepted, being rated from good to excellent by almost the entire population assisted, which would like to repeat it in the future. In regard to the effectiveness of the project, we observed that all the interviewees recalled something from the lessons given, and that the most recalled topics, the risk factors and the benefits of exercise, are issues that should be stressed to increase compliance with exercise ${ }^{15,19}$. In addition, the project had objective results, since $40 \%$ of the participants changed their exercise habits as a function of the lessons given, and $58 \%$ were able to measure their own heart rate. Furthermore, $71 \%$ of the participants reported having passed the information learned to other people.

In conclusion, based on the data gathered, most people visiting the Parque Fernando Costa seem to be there to exercise. Part of these people may be exercising without great risks to their health; some of them (individuals with uncontrolled heart disease, those with symptoms, those with unnoticed high blood pressure), however, may be at a higher risk during exercise, and, therefore, deserve to be referred for medical assessment.

In general, the activity practiced by these people is correct and should benefit health, with a low immediate cardiovascular risk; however, a higher control of intensity is desirable. Despite the knowledge of these people about exercise, we observed ignorance about their own health (use of medication without knowing the reason, presence of symptoms during exercise, ignoring some risk factors, glycemia, serum cholesterol levels, etc.). This suggests the need for campaigns focusing on those subjects.

Based on what was said, we consider that programs, such as that reported in the current study, aimed at assessing the risk of individuals exercising at public places and at providing guidelines for the correct way to exercise and for controlled physical activity, should be stimulated. In fact, each park should have a sector to deal with this subject. Therefore, the population could obtain all the benefits provided by physical exercise without great risk, which would certainly result in a better quality of life for the population and in a reduction in the health costs.

The current absence of such a sector in the parks, however, should not hinder exercise practice in public places, because from the population point of view, the possible risks are by far overcome by the benefits inherent to the regular practice of physical exercise. However, from the individual point of view, individuals with heart disease or with symptoms during exercise should be assessed before beginning their practice.

\section{Acknowledgments}

We thank the management of the Fernando Costa Park for allowing the development of this project and the monitors Cláudio Chaim Rezk, Crivaldo G. Cardoso Jr., Danilo F. Santaella, Marcele A. Coelho, Márcio O. Souza, and Vanessa F. Bisquolo for their collaboration. 


\section{References}

1. Chor D, Fonseca MJM, Andrade CR. Doenças cardiovasculares. Comentários sobre a mortalidade precoce no Brasil. Arq Bras Cardiol 1995; 64: 15-19.

2. Paffenbarger Jr RS, Hade RT, Wing AL, Lee IM, Jung DL, Kampert JB. The association of changes in physical activity and other lifestyle characteristics with mortality among men. NEngl J Med 1993; 328: 538-45.

3. Blair SN, Kohl HW, Paffenbarger Jr RS, Clark DG, Cooper KH, Gibbons LW. Physical fitness and all-cause mortality: a prospective study in health men and women. JAMA 1989; 262: 2395-401.

4. Blair SN, Kohl HW, Barlow CE, Paffenbarger Jr RS, Gibbons LW, Macera CA Changes in physical fitness and all-cause mortality. JAMA 1995; 273: 1093-8.

5. Negrão CE, Forjaz CLM, Rondon MUPB, Brum PC. Adaptações cardiovasculares ao treinamento físico dinâmico. In: SOCESP: Cardiologia, Atualização e Reciclagem. São Paulo: Atheneu, 1996: Cap. 60: 532-40.

6. Tood IC, Wosornu D, Stewart I, Wild T. Cardiac rehabilitation following myocardial infarction; a practical approach. Sports Med 1992; 14: 243-59.

7. Hardman A. Exercise in the prevention of atherosclerotic, metabolic and hypertensive diseases: a review. J Sports Sci. 1996; 14: 201-18.

8. Haapanen N, Miilunpalo S, Vuori I, Oja P, Pasanen M. Association of leisure time physical activity with risk of coronary heart disease, hypertension and diabetes in middle-aged men and women. Int J Epidemiol 1997; 26: 739-47.

9. American College Sports Medicine. ACSM's guidelines for exercise testing and prescription. Baltimore: Williams \& Wilkins, 2000.
10. Rego RA, Berardo FAN, Rodrigues SSR, et al. Fatores de risco para doenças crônicas não-transmissíveis: inquérito domiciliar no município de São Paulo, SP (Brasil). Rev Saúde Pub 1990; 24: 277-85.

11. National Heart Institute. Physical activity and cardiovascular health. JAMA 1996; 276: 241-6.

12. Mittleman MA, Maclure M, Tofler GH, Sherwood JB, Goldberg RJ, Muller JE. Triggering of acute myocardial infarction by heavy physical exertion. $\mathrm{N}$ Engl J Med 1993; 329: 1677-83.

13. American College of Sports Medicine, American Heart Association. Recommendations for cardiovascular screening, staffing, and emergency policies at health/ fitness facilities. Med Sci Sports Exerc 1998; 1009-18.

14. Mion JrD, NobreF.Risco CardiovascularGlobal. SãoPaulo:LemosEditorial, 1999.

15. King AC, Blair SN, Bild DE, et al. Determinants of physical activity and interventions in adults. Med Sci Sports Exerc 1992; 24: S221-S36.

16. Van Camp SP, Peterson RA. Cardiovascular complications of outpatient cardiac rehabilitation programs. JAMA 1986; 256: 1160-3.

17. World Health Organization. Obesity: preventing and managing the global epidemic. Report of a WHO consultation on obesity. Geneva: World Health Organization, 1998.

18. Morris JN, Hardman AE. Walking to health. Sports Med 1997; 23: 306-32.

19. Biddle SJH, Nigg CR. Theories of exercise behavior. Int J Sport Psycol 2000;31: 290-304. 